\title{
Engineering mice to identify autoreactive T-cell antigens
}

$\mathrm{N}$ ow published in the journal Science, Professor Shimon Sakaguchi and colleagues present a novel method of autoantigen discovery and a proof-of-principle, showing that the 60 S ribosomal protein L23a (RPL23A) is an arthritogenic T-cell autoantigen. "By attenuation of T-cell receptor (TCR) signal strength," says Sakaguchi, "T cells recognizing ubiquitous self-antigens, which are normally deleted in the thymus by negative selection, are selected as a dominant repertoire and are causative of systemic autoimmune diseases."

By engineering retrogenic mice to expand and isolate pathogenic TCRexpressing $\mathrm{T}$ cells, a method Sakaguchi says was pioneered by Dr Dario Vignali, the researchers were able to examine a number of individual TCRs for their pathogenicity. Adding to this technique, Sakaguchi says, "We then came up with the idea that by reconstituting arthritisbearing retrogenic mice with $\mathrm{B}$ cells, autoantibodies thus produced by the 'help' of arthritogenic T cells would react with antigens recognized by the arthritogenic TCRs, circumventing other meticulous ways of directly identifying self-peptides."

Whereas autoantibody targets are relatively easy to identify in autoimmune diseases, the autoantigen targets of pathogenic T cells that might be central to rheumatoid arthritis (RA), for example, have been more elusive. Their scarcity in the periphery makes identification of autoreactive $\mathrm{T}$-cell clones and their targets, in patients, a challenge, as $\mathrm{CD}^{+} \mathrm{T}$ cells specific for ubiquitously expressed autoantigens, by definition, have high-affinity TCRs that tag them for thymic deletion.

To overcome these challenges, the researchers expanded pathogenic autoreactive $\mathrm{T}$ cells from SKG mice, a model of spontaneous autoimmune arthritis that is dependent on a mutation of a TCR signalling molecule, tyrosine-protein kinase ZAP-70.

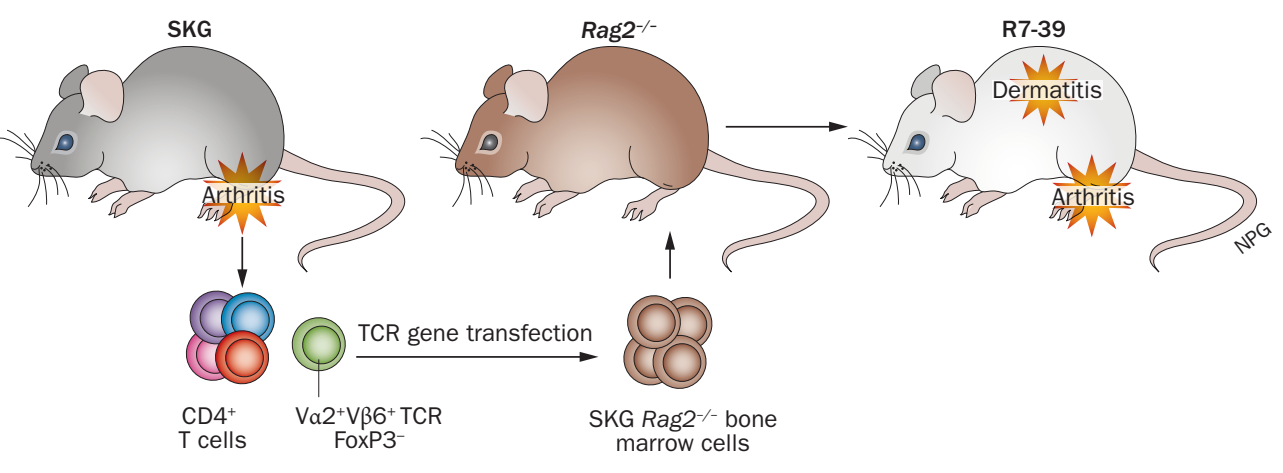

Previously, Sakaguchi's group found that SKG mice have a missense Zap70 mutation (encoding Trp163Cys) that, rather than causing T-cell deficiency, suppresses TCR signalling and thereby alters T-cell ontogeny. As a result, thymocytes with the strongest affinity for self-peptides are positively selected, instead of being deleted. Until now, however, the identity of these autoantigens was unknown.

To identify pathogenic T-cell clones, the researchers knocked-in (to the SKG mice) a fluorescent forkhead box protein $\mathrm{P} 3$ (FoxP3) fusion protein to ensure that protective regulatory $\mathrm{T}$ cells were not selected when isolating $\mathrm{T}$ cells from the arthritic joints of these mice.

Sakaguchi's team then cloned and transfected $\mathrm{Va} 2^{+} \mathrm{V} \beta 6^{+} \mathrm{TCR}$ genes from isolated FoxP3 ${ }^{-} \mathrm{CD}^{+} \mathrm{T}$ cells into bone marrow cells from SKG Rag2 $2^{-/-}$mice (which lack T and B cells). These cells were transferred back into Rag2 $2^{-/-}$mice to generate retrogenic mice with $\mathrm{V} \alpha 2 \mathrm{~V} \beta 6$ restricted thymocytes.

Two arthritogenic TCRs (6-39 and 7-39) and one nonarthritogenic TCR were cloned in this way. $80.0 \%$ of retrogenic 7-39 TCR (R7-39) and $27.3 \%$ of 6-39 TCR (R6-39) mice developed arthritis with mononuclear cell infiltration, pannus formation and cartilage destruction. Furthermore, R7-39 mice developed psoriasis-like dermatitis.

To identify the target of the arthritogenic T cells, Dr Yoshinaga Ito, first author of the study, says, "We reconstituted the retrogenic mice with
$\mathrm{B}$ cells (from TCR $\beta^{-/-}$bone marrow) to generate autoantibodies to the target antigen". Serum from these mice reacted with P3U1 cell extracts and mass spectrometry was used to identify RPL23A as the target, a finding functionally confirmed by stimulating arthritogenic TCR-bearing T-cell hybridomas with

RPL23A is $100 \%$ conserved in mice and humans, and the researchers showed that RPL23A mRNA is highly and ubiquitously expressed in healthy humans. Importantly, they also detected anti-RPL23A humoral and cellular immune responses in patients with RA, but not in patients with osteoarthritis $(n=11)$, systemic lupus erythematosus $(n=30)$, polymyositis or dermatomyositis $(n=10)$, although more patients might be required to see an effect.

Serum anti-RPL23 autoantibodies were detected in a substantially higher proportion of patients with RA $(16.8 \%$, $n=374, P<0.001)$, or with psoriasis $(8.7 \%$, not statistically significant, $n=23)$, than in healthy individuals $(1.3 \%, n=74)$, suggesting RPL23A autoreactivity is pathogenic, at least in a subset of patients.

Of ongoing work, Sakaguchi says, "We plan to further identify other self-antigens with the same method."

Nicholas J. Bernard

Original article Ito, Y. et al. Detection of T cell responses to a ubiquitous cellular protein in autoimmune disease. Science 346, 363-368 (2014) recombinant RPL23A. 